1864 Computer Extracted Features of Nuclear Shape and Architecture Predict Oncotype DX Risk Categories for Early Stage ER+ Breast Cancer

Jon Whitney, Andrew Janowczyk, German Corredor, Hannah Gilmore, Anant Madabhushi. Case Western, Cleveland, $\mathrm{OH}$.

Background: Oncotype DX (ODX) is a 21 gene assay used to stratify women with early stage $\mathrm{ER}+$ breast cancer into low, intermediate and high risk categories, high risk being women who would benefit from adjuvant chemotherapy. In this work we explored the use of computer extracted features of nuclear architecture and morphology from routine $\mathrm{H} \& \mathrm{E}$ images to predict $\mathrm{ODX}$ risk categories for lymph node negative $\mathrm{ER}+\mathrm{BCa}$. Design: The dataset contains $178 \mathrm{BCa}$ patients with H\&E stained whole slide images of low, intermediate, and high ODX risk. Nuclei were segmented and classified as either epithelial or stromal using Deep Learning models, and had nuclear architectural features (median, standard deviation of the edge lengths of nuclear graphs), as well as nuclear shape features extracted.

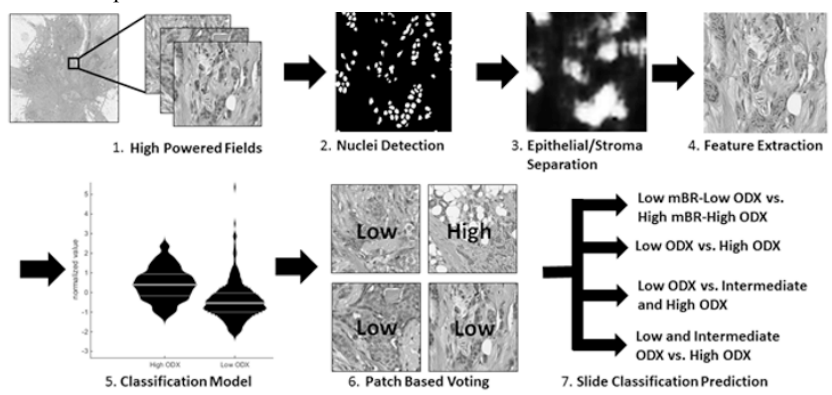

Figure 1. OH ODX Classification Process

6. Patch Based Voting

7. Slide Classification Predictio

These features were used to train a random forest classifier and evaluated via three fold cross-validation. The four experiments we performed were discriminating 1) Low ODX (LODX) and Low modified Bloom-Richardson (mBR) grade vs. High ODX (HODX) and High mBR grade (LL vs. HH), 2) LODX vs. HODX, 3) LODX vs. Intermediate (Int.) and HODX, and 4) LODX \& Int. ODX vs. HODX.

Results: Classification performance was judged for each risk category using Area Under the Curve (AUC) values. The most clearly distinct groups (LL vs. HH) were most consistently classified correctly, with an AUC of 0.77 .

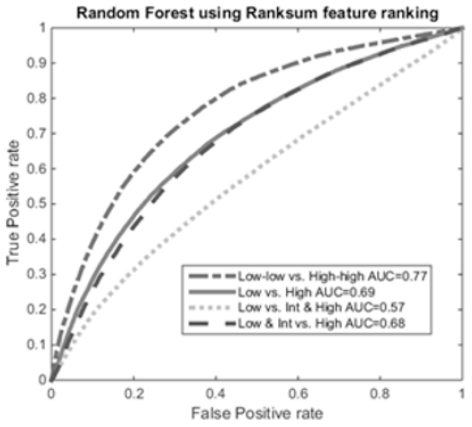

Figure 2. ROC curves for each set of ODX risk categories separated

The highest performing features for separating LODX from HODX were epithelial nuclei architectural features captured by the Cell-Cluster Graph (CCG), shape of stromal nuclei were also found to be important. The standard deviation of CCG Edge Length yielded an $\mathrm{AUC}=0.67$ for separating LODX from HODX.

Conclusions: We showed that computer extracted features of epithelial nuclear architecture and stromal shape from H\&E images can distinguish ODX risk category.

1865 Tissue Based Proteomics on Paraffin Embedded Tissue: A Feasible Means of Identifying and Following Biomarkers in Breast Cancer Richard Wong, Leslie Timpe, Richard Schwab, Farnaz Hasteh, Bruce Macher, Ten-Yang Yen. University of California San Diego, San Diego, CA; UC San Diego, San Diego, CA; San Francisco State University, San Francisco, CA.

Background: The identification and integration of biomarkers into cancer patient care is a cornerstone in the promise of personalized medicine. The evaluation of genetic profiles via sequencing and protein phenotypes via immunohistochemistry are well established protocols in pathology labs. Proteomics by mass spectrometry offers a powerful tool for identifying and following multiple biomarkers, but is not yet integrated into the standard evaluation of patient samples. In this study we establish a protocol with proof of principle for incorporating mass spectrometry into the normal workflow of breast cancer specimens processed into formalin fixed paraffin embedded (FFPE) tissue.

Design: 55 specimens of human FFPE breast tissue were obtained from the Cooperative Human Tissue Network and institutional tissue biorepository. Tissue included breast carcinomas with patient matched normal breast tissue as well as unmatched breast carcinoma samples. For each specimen two 10 micron FFPE sections were cut and treated with xylene and rehydrated. Sections were then incubated in citrate buffer. Trypsin digestion was carried out, proteolytic peptides were recovered, concentrated, and analyzed by LC/MS/MS. Proteins were identified using Mascot, Proteome Discoverer, Scaffold, and Sequest programs.

Results: From all the proteins identified, 290 were selected after being detected in greater than $1 / 3$ of the total samples. Hierarchical clustering analysis showed that profiling these 290 proteins distinguished normal from malignant tissue. Significance analysis further identified 28 proteins that are differentially expressed in the normal vs malignant sapmles. One of these proteins, P11021 (Grp78) has been identified in the literature as a candidate predictive biomarker for response to anthracyclines and platinum based drugs.

Conclusions: Identification and trending of biomarkers is an important part in the management of cancer patients. Genetic and immunophenotypic characterization of tumors have been integrated seamlessly in the current standards of care. The results of this study highlight tissue based proteomics as a practical and feasible means for identifying and following protein biomarkers during the treatment course of a patient's cancer.

1866 A Study of Metastatic Low-Grade Clear Cell Renal Cell Carcinoma by Targeted Next-Generation Sequencing

Sina Zomorrodian, Minghao Zhong, David Y Zhang, Jian Zhuge, Taliya Farooq. New York Medical College at Westchester Medical Center, Valhalla, NY; Icahn School of Medicine at Mount Sinai, New York, NY.

Background: Fuhrman grade is an important criteria in the grading of Clear Cell Renal Cell Carcinoma (CCRCC), however metastasis is still seen in tumors with low clinical grade. As of yet there is no criteria to stratify high risk metastatic CCRCC with indolent types. In this study, we utilize targeted next generation sequencing to study the molecular profile of high risk low grade CCRCC.

Design: Metastatic CCRCC cases were reviewed from 2010 to 2016 on the Softpath database and selected on the basis of low histologic grade (Fuhrman nuclear grade 1 $\& 2$ ) and positivity for metastasis. FFPE tissue was used for targeted next-generation sequencing by the Illumina Truseq Amplicon Panel which targets 48 commonly mutated genes. The genetic variance profile was reviewed for the cases $(n=4)$ and genes containing mutations with a frequency of $\geq 5 \%$ and $<95 \%$ were selected. Selected mutated genes were subsequently reviewed for frequency of occurrence within the cases, and compared with genes listed in a "Comprehensive molecular characterization of clear cell renal cell carcinoma" analyzed by the Cancer Genome Atlas Research Network (CGARN)

Results: Of the mutated genes, 7 were identified in all 4 cases (APC, ATM, FBXW7, GNAQ, KDR, PIK3CA, RET, SMAD4 \& TP53) with freq $=x, 5 \% \leq x<95 \%$. Of these genes, 2 (PIK3CA \& TP53) were previously identified by CGARN.

Conclusions: The genes identified in this study all contained mutations with known deleterious effects. Hence, these genes (both novel and previously reported) are potentially involved in the pathogenesis of metastasis in low-grade CCRCC.

\section{Pediatric Pathology}

1867 Genome-Wide DNA Methylation Profiling in the Diagnosis of Pediatric Ewing Sarcoma, Osteosarcoma, and Synovial Sarcoma Fang Bu, Benjamin Cooper, Peter Wu, Marc Ladanyi, Richard G Gorlick, Matthias Karajannis, Kristen M Thomas, Matija Snuderl. New York University Medical Center, New York, NY; Memorial Sloan Kettering Cancer Center, New York, NY; The Children's Hospital at Montefiore, New York, NY.

Background: Pediatric sarcomas, constituting $15-20 \%$ of pediatric cancers, are a unique diagnostic challenge. Only a subset, including Ewing and synovial sarcomas, harbor pathognomonic genomic alterations. Epigenetic modifications such as changes in DNA methylation are gaining recognition as a primary oncogenic mechanism. Genome-wide methylation profiling could identify biologically and clinically relevant subgroups of tumors, including those without oncogenetic fusions.

Design: We developed a random forest based classifier using the top 400 differently methylated genes for a pilot cohort $(\mathrm{N}=36)$ of three common pediatric bone and soft tissue sarcomas. Using the Illumina Infinium Human Methylation450 BeadChip Array (450K array) platform, we performed genome-wide DNA methylation analysis on 10 Ewing sarcomas, 15 osteosarcomas, and 11 synovial sarcomas, including formalinfixed paraffin-embedded, frozen, and fresh tissue. Unsupervised hierarchical clustering analysis was performed to compare methylation signatures among tumor types.

Results: On molecular studies, 6 of 11 synovial sarcomas had an SYT fusion and all 10 Ewing sarcomas had an EWSR/FLI1 fusion. Unsupervised hierarchical clustering analysis showed distinct DNA methylation profiles for each sarcoma type. Synovial sarcomas clustered together, EWSR fusion sarcomas made a second cluster, and osteosarcomas formed a third. We validated our classifier against publicly available methylation data from the TCGA-SARC and TARGET-OS databases for synovial and osteosarcomas, respectively, and obtained $98.9 \%$ concordance.

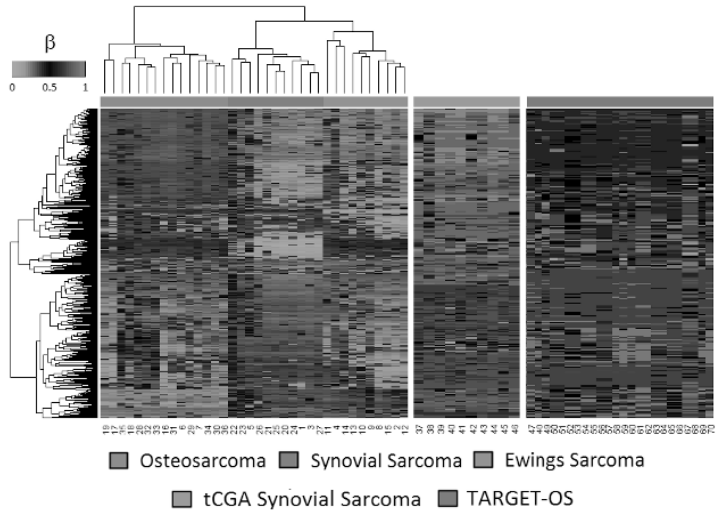


Conclusions: We were able to show distinct epigenetic signatures for three common pediatric sarcoma types and to develop a classifier based on the top differentially methylated genes. Our results suggest a role for DNA methylation profiling in their diagnosis, particularly in tumors lacking pathognomonic fusions or with unknown mutation status.

\section{YY1 EXPRESSION IN WILMS-' TUMOR}

Lourdes Cabrera-Muñoz, Sara Huerta-Yepez, Luis E Juarez-Villegas, Marta ZapataTarres, Stanislaw Sadowinski-Pine. Hospital Infantil de Mexico Federico Gomez, Mexico, Mexico; Hosptal Infantil de Mexico Federico Gomez, Mexico, Mexico. Background: The transcription factor Ying-Yang 1 (YY1) has an important regulatory role in tumorigenesis; high YY1 nuclear protein expression are detected in cell lines and tumors from several genitourinary carcinomas, including Renal Cell Carcinoma; YY1 expression in Wilms' tumor (WT) has not been reported. The aim of this study was to investigate the expression of YY1 in WT and the potential relationships with clinicopathological features.

Design: Using tissue microarrays (TMA), expression of YY1 protein was evaluated by immunohistochemistry in 57 cases of WT. Archived formalin-fixed paraffin-embedded tissue from tumors and non tumoral renal tissue from the same patient was used for TMA and stained with polyclonal antibody against YY1. Immunohistochemical expression level was determined semiquatitative by two pathologists and quantitatively by densitometry. Both criteria matched and samples scored as weak $1+$, moderate $2+$, or strong $3+$ and results correlated with clinicopathologic features.

Results: There were 26 males and 31 females, ages ranged at diagnosis from 9-111 months (mean 39.5 months). Tumors were unilateral in 54 cases: 31 (57.4\%) right, 23 (42.5\%) left and bilateral in 3 cases, Favorable Histology (FH) 54 (94.7\%). Unfavorable Histology (UH) $3(5.3 \%)$, patients clinical stage were: stage I, 8 (14.0\%), II, 17 (29.8\%), III $22(38.6 \%)$, IV, 7 (12.3\%), V, $3(5.3 \%)$. YY1 nuclear and cytoplasmic expression was present in non-tumoral tubular cells and WT; higher levels were observed in epithelial, blastemal and mesenchymal WT components, been more frequently expressed in blastemal cells $(\mathrm{p}=0.001, \mathrm{r}=0.343)$. After stratifiying by $\mathrm{YY} 1$ protein expression level, 12 patients (21\%) had YY1 strong expression, 11 FH WT and 1 UH WT, from these, $4(30 \%)$ patients showed a WT with predominantly blastemal component. YY1 overexpession was related with a higher $(>2.5)$ risk of adverse event (local recurrence and lung an liver metastasis at 200 weeks follow up) and slightly correlate with decreased survival $(\mathrm{p}=0.02)$. YY1 protein levels were independent of patient age, clinical stage and tumor histology.

Conclusions: Our results show thatlevels ofYY1 protein expression are significantly elevated in WT.

Higher levels of YY1 expression were associated with increased risk of tumor recurrence and distant metastasis its also may be related to decreased survival of patients.

\section{Clear Cell Sarcoma of Kidney Is Characterized by BCOR Gene Abnormalities Including Exon 16 Internal Tandem Duplications and BCOR- CCNB3 Gene Fusion}

Kenneth Chang, Meng Kang Wong, Sze Jet Aw, Cedric Ng, Vikneswari Rajasegaran, Derrick Lian, Chik Hong Kuick, Jain Sudhanshi, Eva Loh, Amos Loh, Minzhi Yin, Angela Goytain, Tony Ng, Bin Tean Teh. KK Women's and Children's Hospital, Singapore, Singapore; National Cancer Centre Singapore, Singapore, Singapore; Shanghai Children's Medical Centre, Shanghai, China; University of British Columbia, Vancouver, Canada; Vancouver General Hospital, Vancouver, Canada; Duke-NUS Medical School, Singapore, Singapore.

Background: Clear cell sarcoma of kidney (CCSK) is a rare pediatric renal malignant tumor. A majority of CCSKs have internal tandem duplications (ITD) of the $B C O R$ gene. A minority have the YWHAE-NUTM2 gene fusion. A third 'double-negative' (DN) category comprises CCSKs without BCOR ITD or YWHAE-NUTM2 fusion.

Design: We characterized 12 CCSKs histologically, immunohistochemically (with cyclin D1, BCOR and CCNB3 stains), and genetically. Gene fusions were identified by RT-PCR, an in-house NanoString $(\mathbb{R}$-based assay, and a next-generation sequencingbased anchored multiplex PCR (AMP) technique. We also Sanger sequenced the $B C O R$ ITD region, and performed next-generation sequencing of the entire BCOR gene. Results: All 12 CCSKs had typical histomorphology. There was strong diffuse cyclin D1 immunoreactivity in 11 of 11 cases tested. 9 cases (75\%) had BCOR exon 16 ITD, and BCOR immunohistochemistry was strong, weak and negative in 1, 6 and 2 cases respectively. None had the YWHAE-NUTM2 fusion. Among the $3 \mathrm{DN}$ cases $(25 \%)$, one had a $B C O R-C C N B 3$ fusion detected by the NanoString ${ }^{\circledR}$ assay, and strong nuclear CCNB3 but negative BCOR immunoreactivity. Quantitative PCR showed markedly elevated BCOR gene expression in this case whereas $B C O R$ ITD cases had lower levels. A second DN case had an unusual BCOR staining pattern of strong immunoreactivity in $5 \%$ of tumour cells but no identifiable $B C O R$ gene alteration. The third DN case had no identifiable gene fusion or $B C O R$ gene alteration.

Conclusions: The majority of these CCSKs have BCOR ITD, and none have the $Y W H A E-N U T M 2$ gene fusion. We verify the strong, diffuse cyclin D1 immunoreactivity in CCSKs described in recent reports. BCOR immunoreactivity is not consistently strong in all CCSKs with $B C O R$ ITD and cannot be used as a diagnostic immunostain to identify $B C O R$ ITD cases. Amongst the DN cases is a $B C O R$ - $C C N B 3$ sarcoma. Typically a bone sarcoma affecting male adolescents, this is the first report of a $B C O R-C C N B 3$ sarcoma presenting in a kidney of a young child as a CCSK. The full spectrum of DN CCSKs awaits fuller characterization.
1870 MDR3 and MRP1 Overexpression Are Poor Prognosis Markers in Wilms Tumor Patients After SIOP-93-01 Standard Treatment Protocol Enrique de Alava, Lourdes Hontecillas, Daniel J Garcia-Dominguez, Diego PascualVaca Gumez, Rosa García-Mejias, Gema L Ramirez-Villar, Carmen Saez. Instituto de Biomedicina de Sevilla (IBiS), Hospital Universitario Virgen del Rocío/CSIC/ Universidad de Sevilla, Seville, Spain; Pediatric Oncology Unit, Hospital Universitario Virgen del Rocío, Sevilla, Seville, Spain.

Background: Wilms tumor (WT) is the most common renal tumor in children. Most WT patients respond to chemotherapy. However, a subset of tumors fails to respond to chemotherapy. Resistance to chemotherapy remains the major obstacle to a successful therapy. The development of chemoresistance in a variety of neoplasms seems related to overexpression of multidrug resistant (MDR) transporters. The role of MDR transporters in WT is not well characterized. Thus, we aimed to identify molecular prognostic markers by characterization of expression profiles of MDR genes in WT patient samples. Design: This retrospective study included two different sample series obtained between 1993 and 2006 which corresponded with WT patients treated by neoadjuvant chemotherapy before nephrectomy according to the SIOP-93-01 standard protocol. Series 1 comprised 42 kidneys and 46 WT frozen samples ( 40 paired samples). Series 2 comprised a Tissue Microarray (TMA) with samples from $31 \mathrm{WT}$ and 9 normal kidney patients. The expression of nine MDR transporters (BCRP1 (breast cancer resistance protein), MDR1 (p glycoprotein), MDR3 (Multidrug resistance 3), MRP1 (Multidrug resistance-associated protein 1),MRP2,MRP3,MVP1 (Major Vault protein), $\mathrm{VPARP}$ and TEP1) was evaluated in Series 1 by RT-PCR and Western blot. MDR3 and MRP1 expression was evaluated also by immunohistochemistry in Series 2 . The results obtained in both analyses were correlated with clinical parameters.

Results: The majority of the studied MDR transporters (6/9) was downregulated or unchanged in tumor samples with respect to control tissues. However, BCRP1, MDR3 and MRP1 were upregulated in tumors versus control tissues. MDR3 and MRP1 overexpression correlated with high risk ( $p$ value $<0.0001$ and $p$ value $<0.0001$ respectively) and blastemal subtype ( $\mathrm{p}$ value $<0.001$ and $\mathrm{p}$ value $<0.01$ respectively). The disease free survival was significantly shorter in patients with high MDR3 transcript levels ( $p$ value $=0.0359$ ). Also, we evaluated the blastemal WT tumors and we observed that low expression of MDR3 in blastemal subtype tumors was associated with a good prognosis in this high-risk subset $(\mathrm{p}=0.0328)$.

Conclusions: MDR3 and MRP1 could play a role in drug resistance in Wilms tumor treatment, probably by alteration of an unspecific drug excretion system. In addition, we have described MDR3 as a new biomarker for stratification of patients with blastemal subtype Wlims tumor.

\section{Identification of 2 Novel Transcript Fusions Coexisting with} EML4-ALK in a New Case of ALK-Positive Pediatric Renal Cell Carcinoma Larisa Debelenko, Huang Weihua, Jian Zhuge, Changhong Yin, Minghao Zhong, Mehmet Ozkaynak, John T Fallon. New York Medical College, Valhalla, NY.

Background: ALK-rearranged renal cell carcinoma (RCC) is rare, with only 12 cases reported in children and adults. Susceptibility of ALK+ RCC to targeted therapies is not known and ALK inhibitor Crizotinib has not been approved by FDA for non-lung ALK+ tumors thus far.

Design: Tissue from a recurrent RCC in a 15-year old was collected according to the New York Medical College IRB-approved protocol, with the patient's guardian informed consent. ALK antibody D5F3 (Ventana) and Vysis ALK Break Apart FISH Probe (Abbott Molecular) were used. The tumor and normal tissue RNAs were subject to next-generation sequencing (NGS) using TruSeq stranded mRNA preparation kit and paired-end sequencing $(76 \mathrm{bp} \times 2)$ (Illumina). TopHat and Manta fusion algorithms were utilized. The gene fusions were validated by RT-PCR with appropriate controls, followed by Sanger sequencing. EML4-ALK fusion was independently confirmed by Lung Cancer Comprehensive Translocation and Mutation Panel at ARUP.

Results: The primary tumor was initially diagnosed as RCC, not otherwise specified, due to unusual high grade histology. ALK studies showed diffuse cytoplasmic staining by IHC and rearrangement by FISH. NGS analysis indicated $E M L 4$ as the $A L K$ fusion partner and, unexpectedly, showed similarly high scores for $H S F 1-S P O N 1$ and $M L X I P L$ GLCCII fusions, not reported previously in any neoplasia. RT-PCR with primers flanking putative breakpoints and subsequent sequencing confirmed exon 1 of $H S F I$ fused to exon 7 of SPON1 and exon 2 of MLXIPL followed by exon 2 of GLCCII, predicting 572 aa and 528 aa fusion oncoproteins, respectively. NGS also identified missense mutations of TP53 and POLE genes, but no point mutations associated with drug-resistance in the $A L K$ kinase domain. The patient was briefly treated with Crizotinib off-label, with no success as demonstrated by massive recurrence. Pretreatment tumor showed complex near-tetraploid karyotype with multiple structural and numerical abnormalities, which only partially correlated with detected fusions.

Conclusions: We report the $13^{\text {th }}$ case of ALK + RCC. NGS in combination with tumor chromosome analysis demonstrated simultaneous multiple genomic alterations, including at least 3 fusion transcripts, consistent with chromothrypsis as a mechanism underlying this ALK + tumor. Multiple fusion transcripts in a single tumor demonstrate inaccuracy of tumor classification based on a single associated translocation and question the efficacy of treatment with a single target agent.

\section{Clinical, Microscopic and Molecular Presentation of Pediatric} Cutaneous Leishmaniasis

Gabriel Dunya, Ibrahim Khalifeh. American University of Beirut, Beirut, Lebanon. Background: Cutaneous leishmaniasis (CL) is an emerging uncontrolled tropical parasitic disease in endemic and nonendemic areas with high prevalance in pediatric group.

Design: 382 Individuals from Lebanon, Saudi Arabia, Pakistan and Syria diagnosed with CL by punch biopsy/scrapings were grouped into adults ( $>18$ years) or Pediatrics $(\leq 18$ 
years). Data recorded includes clinical [number, location, type, size and extensivenes (size larger than $3 \mathrm{~cm}$ of largest lesion, more than 5 lesions per patient, lesion presen for more than 12 months, disfiguring lesion or closeness to vital sensory organs) of lesions], microscopic [Ridley's Parasitic Index and Pattern] findings. In addition, molecular confirmation and speciation were performed.

Results: In comparison to adults, Patients in the pediatric group ( $\mathrm{n}=158,41.4 \%)$ showed significantly higher number of lesions, more facial involvement, and more extensive disease $(\mathrm{P}<0.05)$. Microscopically, more advanced Ridley's pattern has been observed. The other variables didn't show statistical difference between the two groups.

\begin{tabular}{|c|c|c|c|c|}
\hline \multirow{2}{*}{\multicolumn{2}{|c|}{ Count }} & Adult & Pediatric & P Value \\
\hline & & $224(58.6 \%)$ & $158(41.4 \%)$ & \\
\hline \multirow{2}{*}{ Gender } & Male & $118(52.7 \%)$ & $92(58.2 \%)$ & \multirow{2}{*}{0.28} \\
\hline & Female & $106(47.3 \%)$ & $66(41.8 \%)$ & \\
\hline \multicolumn{2}{|c|}{ Average Lesion duration (months) } & $7.77 \pm 11.8$ & $6.53 \pm 6.61$ & 0.23 \\
\hline \multicolumn{2}{|c|}{ Average Number of lesion* } & $2.01 \pm 1.95$ & $2.69 \pm 3.23$ & 0.01 \\
\hline \multicolumn{2}{|c|}{ Extensive Disease* } & $70(31.3 \%)$ & $84(53.2 \%)$ & 0.0001 \\
\hline \multirow{3}{*}{ Parasitic Index } & Low & $108(48.4 \%)$ & $69(45.7 \%)$ & \multirow{3}{*}{0.78} \\
\hline & Intermediate & $33(14.8 \%)$ & $21(13.9 \%)$ & \\
\hline & High & $82(36.8 \%)$ & $61(40.4 \%)$ & \\
\hline \multirow{2}{*}{ Ridely's Pattern* } & Early & $116(53.0 \%)$ & $56(39.7 \%)$ & \multirow{2}{*}{0.01} \\
\hline & Advanced & $103(47.0 \%)$ & $85(60.3 \%)$ & \\
\hline \multirow{2}{*}{ Molecular } & L. Tropica & $202(90.2 \%)$ & $147(93.0 \%)$ & \multirow{2}{*}{0.43} \\
\hline & L. Major & $22(9.8 \%)$ & $11(7.0 \%)$ & \\
\hline
\end{tabular}
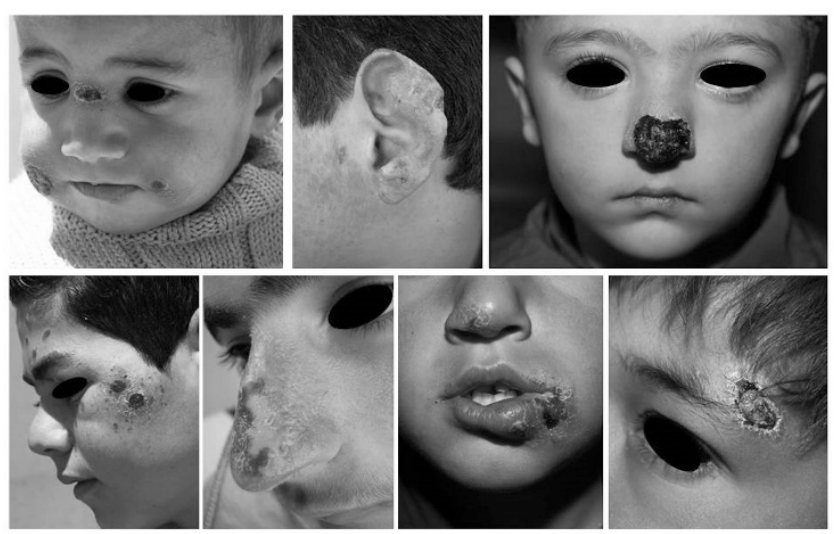

Conclusions: Historically, CL has been known to be a neglected tropical disease of poverty and pediatric predilection. In our pediatric group, CL shows more extensive disease clinically mirrored by more advanced lesions microscopically.

1873 Deep Juvenile Xanthogranuloma (DJXG), 41 New Subcutaneous (SQ) and Intramuscular (IM) Cases, Reclassification as a Benign Fibrohistiocytic Tumor Variant

Julie Fanburg-Smith, Michele Anderson, Katja Specht, Aaron Auerbach, Brian Rubin. National Academic Pathology, McLean, VA; AFIP/Joint Pathology Center, Silver Spring, $\mathrm{MD}$; Cleveland Clinic, Cleveland, $\mathrm{OH}$.

Background: Cutaneous juvenile xanthogranuloma (JXG) has been considered among the benign epithelioid to spindled histiocytic-phenotype lesions of the dermis, usually in the head and neck of infants. So called "deep" JXG has been confused with extracutaneous visceral lesions. We wanted to reclassify and redefine a large group of "deep" JXG.

Design: Archival cases coded as "JXG" were pulled and analyzed from the authors files, excluding dermal and visceral tumors.

Results: There were 30 intramuscular (IM) and 21 subcutaneous (SQ) JXG in 28 males, 20 females and 1 unknown gender, all but two cases in less than one-year old infants. Sizes ranged from $0.5-9.0 \mathrm{~cm}$, IM twice as large as SQ. Locations included proximal extremity $(n=13)$, trunk $(n=30)$, and head and neck $(n=6)$, with IM more proximal than SQ. There were no associated syndromes/clinical findings identified. All cases were composed of plump histiocytoid cells arranged in sheets without distinct cell borders, non-prominent nucleoli, and few normal form mitoses, only one case with focal necrosis. IM had infiltrative growth retaining skeletal muscle fibers and crossing fascial planes. SQ were well-delineated and ball-like. Touton-type giant cells, xanthoma cells, and eosinophils were minimal compared with classic cutaneous variant. Immunostains revealed at-least focally positive SMA, Factor XIIIa, CD163, KP-1, and lysozyme and negative $\mathrm{S} 100$ protein, CD21, CD35, and CD1a. No patients died of disease, with up to 23 years follow-up.

Conclusions: Deep JXG is usually a truncal pediatric tumor defined as SQ or IM, with aberrant reversed infiltration of skeletal muscle or ball like pattern in subcutis. Phenotypically "fibrohistiocytic," these can be separated from histiocytic and giant cell rich tumors by cytology, relative-paucity of eosinophils, Touton-giant cells and xanthoma cells, nested SQ and fascial-infiltrative IM patterns, and immunophenotype. Deep JXG is larger, more proximal on trunk, with less eosinophils than classic cutaneous JXG counterparts. While the deep location, large size and unusual proximal limb and trunk location with a few mitoses in DJXG may be worrisome in an infant, these deep JXG can be recognized by reproducible immuno-morphologic, growth, and clinical features and have benign outcome.
1874 The Role of Hypoxia-Inducible NPY System in Dissemination of Ewing Sarcoma - A Mouse Xenograft Model

Susana Galli, Jason Tilan, Sung-Hyeok Hong, Rachel Acree, Katherine Connors, Meredith Horton, Akanksha Mahajan, Larissa Wietlisbach, Yi-Chien Lee, Olga Rodriguez, Christopher Albanese, Joanna Kitlinska. Georgetown University Medical Center, Washington, DC.

Background: Ewing sarcoma (ES) is a tumor of children and young adults that develops in bones and soft tissues. The presence of metastases is the most adverse prognostic factor, yet the mechanisms governing their formation remain unclear. A transcriptional target of EWS-FLI1, neuropeptide Y (NPY), is highly expressed and released from ES tumors. Hypoxic tumor environment further increases NPY expression and activates its pro-metastatic functions, suggesting the role for NPY in ES dissemination. Here, we tested the effect of primary tumor hypoxia on NPY system expression and the resulting metastases in a mouse xenograft model.

Design: ES cell lines, SK-ES1 and TC71, with high and low peptide release respectively, were injected into gastrocnemius muscles of SCID/beige mice. To determine if the metastatic pattern changes under hypoxic conditions, the mice were subjected to femoral artery ligation (FAL). The location, frequency and latency of metastases were compared between mice bearing FAL-treated and control tumors.

Results: FAL-induced hypoxia resulted in marked necrosis in ES primary tumors. Nevertheless, immunostaining for NPY and its receptors, Y2R and Y5R, was increased in areas of hypoxic, yet still viable primary tumor tissue. This increase in NPY expression was associated with elevated NPY levels in plasma of mice bearing FAL-treated primary tumors. In FAL-treated SK-ES1 xenografts, primary tumor hypoxia resulted in increase in number of metastases and decrease in their latency. The most profound increase was observed in metastases to various internal organs. Hypoxic TC71 xenografts presented with frequent bone metastases, which were not observed under basal conditions. Our previous data indicated involvement of NPY in ES bone invasion. Thus, the increase in osseous metastases observed in mice bearing FAL-treated TC71 xenografts may be triggered by hypoxia-induced up-regulation of NPY release in this normally NPY-low cell line. Importantly, the pattern of metastases observed in mice bearing hypoxic primary tumors resembles this observed in patients with highly necrotic ES.

Conclusions: Hypoxia exacerbates ES metastasis and promotes dissemination to internal organs and bones. NPY system activated by hypoxia is a candidate mediator of its pro-metastatic actions. Our model of tumor hypoxia in vivo provides an excellent opportunity to identify pathways involved in ES metastatic progression that subsequently may become novel therapeutic targets for this disease.

1875 Definition of a 3-Protein Signature to Stratify Hepatoblastoma Patients According to Their Prognosis

Marta Garrido, Laura Guerra, Marina Simon, Mikel Azkargorta, Lara Nonell, Manuel Lopez-Santamaria, Maria E Mateos, Cristina Belendez, Diego Plaza, Francisco Hernandez, Marta Mendiola, Isabel Ojanguren, Margaret Childs, Piotr Czauderna, Rudolf Maibach, Bruce Morland, Marie A Buendia, Felix Elortza, Ramon Planas, Maria $R$ Sarrias, Margarita Sala, Carolina Armengol. Hospital Vall d'Hebron, Barcelona, Spain; Hospital La Paz, Madrid, Spain; HealthSciences Research Institute Germans Trias i Pujol, Badalona, Spain; CIBEREHD, Barcelona, Spain; CIC bioGUNE-ProteoRedISCIII, Derio, Spain; IMIM, Barcelona, Spain; Hospital Reina Sofia, Cordoba, Spain; Hospital Gregorio Marañón, Madrid, Spain; Hospital Germans Trias i Pujol, Badalona, Spain; Nottingham Clinical Trials Unit, Nottingham, United Kingdom; Medical University of Gdansk, Gdansk, Poland; IBCSG, Berne, Switzerland; Birmingham Children's Hospital, Birmingham, United Kingdom; Centre Hepatobiliaire Paul Brousse, Villejuif, France; IGTP, Badalona, Spain.

Background: Hepatoblastoma (HB) is the main liver tumor in childhood. Curative treatment is possible by combining chemotherapy and surgery. Nevertheless, $20 \%$ of HB patients do not survive cancer and survivors can be affected by serious side effects related to chemotherapy. Current clinical stratification relies only on clinical and pathological parameters. The aim of this study was to identify new protein biomarkers of the 2 previously identified $\mathrm{HB}$ prognostic subtypes, $\mathrm{C} 1$ and $\mathrm{C} 2$, in order to improve patient stratification.

Design: A total of 122 pediatric patients with HB (mean age $2.2 \mathrm{yr}$, sera AFP range: $150-7.627 .330 \mathrm{ng} / \mathrm{ml}, 19$ with metastasis, mean follow-up: 64.8 months, 16 deaths) were included. Sixteen of them $(\mathrm{C} 1, \mathrm{n}=11 ; \mathrm{C} 2, \mathrm{n}=5)$ and 8 normal liver $(\mathrm{NL})$ were analyzed by two proteomic techniques: 2D-Difference Gel Electrophoresis (2D-DIGE) and Label-Free (LF) nLC-MS/MS. Prognostic biomarkers were identified by supervised analysis comparing the proteomic profiling of $\mathrm{HB}$ subtypes and using NL as a reference and validated by Western Blot (WB) and immunohistochemistry (IHC) in 106 cases. Results: Eight new biomarkers were selected $(\mathrm{FC} \pm 2, \mathrm{p}<0.01)$ among 411 differently expressed proteins and their expression evaluated by WB. Three of them were further validated by IHC. The expression changes of the biomarker combination showed a strong association with patient event free survival (EFS) $(\log$ rank $=0.008)$. This protein signature applied together with the current clinical stratification showed a higher association with EFS ( $\log$ rank $=0.003)$.

Conclusions: Through a proteomic study, we have defined a 3-protein prognostic signature that is easily applicable to clinical practice and improve current patient stratification of pediatric patients with liver cancer.

\section{PD-L1 Expression in Villitis of Unknown Etiology}

Daniel L Geisler, Lisa Friedman, Margaret Moore, Anne Mills, Robin LeGallo. University of Virginia, Charlottesville, VA.

Background: Villitis of unknown etiology is a maternal immunologic attack of placental villi, typically in the third trimester. When severe, it is associated with adverse fetal outcome and significant risk of recurrence. It is thought to result from the break-down of the normal maternal-fetal tolerance. The inflammatory infiltrate is comprised predominantly of maternal T-lymphocytes and macrophages. Programmed 
cell death receptor ligand (PD-L1) is an immune checkpoint that downregulates the inflammatory response, permitting self-tolerance and mitigating against autoimmunity. Its role in fetal tolerance is less clear. PD-L1 can be expressed by both inflammatory and non-inflammatory cells. PD-L1 is strongly expressed on the surface of the syncytiotrophoblasts in normal chorionic villi and a lesser degree in the intermediate trophoblasts; all other cell types are negative. We therefore speculated that deficient or aberrant PD-L1 expression may be observed in the villi affected by villitis.

Design: Surgical pathology database was queried for cases of chronic villitis and reviewed for to confirm diagnosis and grade of villitis. Sections were stained using an antibody directed against PD-L1 (Spring Bioscience SP142). The slides were examined for expression of PD-L1 on the villous surface and maternal inflammatory cells.

Results: Five cases of low-grade villitis and five cases of high-grade villitis, two of which has evidence of vascular damage, were examined as well as a normal third trimester placenta as a control. All cases showed the typical PD-L1 pattern in noninflamed villi. In the villi affected by villitis, PD-L1 was retained on the surface of the villi. In addition, the villous stromal and Hofbauer cells were PD-L1 positive. The maternal inflammatory response also showed strong and diffuse staining in all villitis cases.

Conclusions: PD-L1 expression is not reduced within the villous surface in placentas with chronic villitis of unknown etiology, providing no support for a maternal inflammatory attack directly related to aberrant PD-L1 expression by fetal villi. Rather, PD-L1 staining is increased within the villous stroma in villi affected by villitis. The presence of PD-L1 positivity within affected villi suggests that there is an ongoing attempt to curb the inflammatory response.

1877 Malignant Ectomesenchymoma(MEM): Series Analysis of a Histologically and Genetically Heterogeneous Tumor

Brannan Griffin, Pauline Chou, David George, Lawrence Jennings, Nicoleta Arva. Northwestern University, Feinberg School of Medicine, Chicago, IL; Lurie Children's Hospital of Chicago, Chicago, IL.

Background: MEM is a rare neoplasm with dual malignant mesenchymal and neuroectodermal elements. Mesenchymal elements are usually rhabdomyosarcoma (particularly embryonal) and neuroectodermal derivatives often neuroblastic tumor. MEM manifests in various sites given wide neural crest cell migration. No unique identifying molecular abnormality is known. We analyzed the spectrum of MEM seen in our population.

Design: Retrospective study identified 5 patients diagnosed with MEM. Data obtained included age, sex, tumor size and site, histologic findings, immunophenotypic profile, molecular analysis by RT-PCR or NGS, treatment and outcome.

Results: See table.

\begin{tabular}{|c|c|c|c|c|c|c|}
\hline Age & Sex & Site & Size & Histology & $\begin{array}{c}\text { Molecular } \\
\text { Findings }\end{array}$ & $\begin{array}{l}\text { Treatment/ } \\
\text { Outcome }\end{array}$ \\
\hline $4 y$ & F & Axilla & N/A & $\begin{array}{l}\text { ARMS and Neuroblasto- } \\
\text { maARMS (desmin, myo- } \\
\text { genin+) with intermixed } \\
\text { cells showing neuronal } \\
\text { differentiation (tyrosine } \\
\text { hydroxylase(TH)+, CD99-) }\end{array}$ & $\begin{array}{c}\text { PAX3- } \\
\text { FOXO1 (RT- } \\
\text { PCR) }\end{array}$ & N/A \\
\hline $4 \mathrm{M}$ & $\mathrm{F}$ & Neck & $\begin{array}{c}6 \\
\mathrm{~cm}\end{array}$ & $\begin{array}{l}\text { ERMS and PNETPNET } \\
\text { (CD99, synaptophysin+) } \\
\text { with intermixed ERMS } \\
\text { (spindle cells with rhabdo- } \\
\text { myoblastic differentiation) }\end{array}$ & $\begin{array}{l}\text { AZF1-ATRX } \\
\text { (NGS) }\end{array}$ & $\begin{array}{l}\text { PNET proto- } \\
\text { col; deceased } \\
\text { at } 8 \text { months } \\
\text { from brain } \\
\text { metastasis }\end{array}$ \\
\hline $\begin{array}{c}15 \\
\mathrm{y}\end{array}$ & M & $\begin{array}{c}\text { Parates- } \\
\text { ticular }\end{array}$ & $\begin{array}{c}4 \\
\mathrm{~cm}\end{array}$ & $\begin{array}{l}\text { ERMS and } \\
\text { Ganglioneuroma(GN)ERMS } \\
\text { (fascicles of spindle cells; } \\
\text { myogenin, desmin }+ \text { ) inter- } \\
\text { mixed with ganglion cells } \\
\text { associated with schwannian } \\
\text { stroma }\end{array}$ & $\begin{array}{l}\text { No alteration/ } \\
\text { fusion detect- } \\
\text { ed (NGS) }\end{array}$ & $\begin{array}{c}\text { COG } \\
\text { ARST0331 } \\
\text { RMS proto- } \\
\text { col; no disease } \\
(15 \text { months })\end{array}$ \\
\hline $1 \mathrm{M}$ & $\mathrm{F}$ & $\begin{array}{l}\text { Perine- } \\
\text { um }\end{array}$ & $\begin{array}{c}5 \\
\mathrm{~cm}\end{array}$ & $\begin{array}{l}\text { ERMS and GNERMS } \\
\text { (fascicles of spindle cells; } \\
\text { myogenin, desmint) inter- } \\
\text { mixed with ganglion cells } \\
\text { associated with schwannian } \\
\text { stroma }\end{array}$ & N/A & $\begin{array}{c}\text { COG } \\
\text { ARST0331 } \\
\text { RMS proto- } \\
\text { col; alive at } \\
20 \text { months; } \\
\text { disease status } \\
\text { unknown } \\
\end{array}$ \\
\hline $6 \mathrm{M}$ & $\mathrm{F}$ & Neck & $\begin{array}{c}2 \\
\mathrm{~cm}\end{array}$ & $\begin{array}{l}\text { ARMS and PNETARMS } \\
\text { (myogenin, desmin+) with } \\
\text { intermixed neuropil and cells } \\
\text { forming rosettes (CD99+, } \\
\text { TH-) }\end{array}$ & $\begin{array}{c}\text { PAX7- } \\
\text { FOXO1 (RT- } \\
\text { PCR) }\end{array}$ & $\begin{array}{c}\text { alveolar RMS } \\
\text { protocol; alive } \\
\text { at } 16 \text { months; } \\
\text { disease status } \\
\text { unknown }\end{array}$ \\
\hline
\end{tabular}

Conclusions: One of the largest, our analysis extends the heterogeneous histologic and molecular spectrum. Mesenchymal elements, RMS, were dominant in $4 / 5$ cases. $2 / 5$ cases with ARMS also harbored subtype-characteristic translocations. PNET was the neuroectodermal component in $2 / 5$ cases, suggesting this element may be underreported. Novel JAZF1-ATRX gene fusion product was seen in one case, but no unifying alterations were found. Derivatives demonstrating second line of differentiation were scant, stressing a high degree of suspicion and thorough workup required for accurate diagnosis.
1878 Familial Acute Myeloid Leukemia/Myelodysplastic Syndrome with Germline RUNX1 Mutations: A Single Instituion Experience

Vivian Hathuc, Kelly Walkovich, Dale Bixby, Lina Shao, Lauren Smith. University of Michigan, Ann Arbor, MI.

Background: Acute myleoid leukemia (AML) and myelodysplastic syndromes (MDS) associated with germline mutations are rare. In an era of next-generation sequencing, these mutations are becoming more identifiable. An understanding and recognititon of these patients is important for treatment planning, genetic counseling, and long-term outcome. Little has been published on the pathological features of patients with AML/ MDS and germline RUNXI mutations.

Design: Patients with familial AML/MDS were identified in our databases. Two patients were identified with germline $R U N X 1$ mutations. Four patients were identified with GATA2 mutations. Three patients were identified with dyskeratosis congenita. The morphologic and immunophenotypic features were studied in the patients with RUNXI mutations and these were compared to findings in other types of familial AML/MDS. Results: The patients with $R U N X 1$ germline mutations were a 14-year-old boy and a 37-year-old woman with congenital neutropenia. Morphologically, both patients had blasts with prominent azurophilic cytoplasmic granules. Based on flow cytometric immunophenotyping, both patients had blasts with expression of CD19 (dim). However, interestingly, the immunophentoype shifted over the course of disease in both patients, with CD19 expression being variable from study to study. In addition, one of the patients had CD7 expression which remained constant and the other had CD56 expression which also remained constant throughout the disease. The 14-year-old boy died of refractory AML status/post bone marrow transplant. The 37 -year-old woman is 500 days staus/ post-transplant with no evidence of disease.

Conclusions: Patients with germline $R U N X I$ mutations may show antigenic shift of the flow cytometric immunophenotype over the course of the disease. CD19 expression, if seen, may be helpful in initially determining whether $R U N X I$ germline testing could be beneficial. As this syndrome is very rare, additional patients with germline $R U N X I$ mutations will need to be studied to determine whether these observations are robust.

\section{Pulmonary Alveolar Proteinosis Associated with STAT5b} Deficiency: A Rare Entity

John Hicks, David Berger. Texas Children, Houston, TX.

Background: STAT5b is a transcription factor that mediates signal transduction associated with IL2, IL4, CSF1 and growth hormones. It is involved in TCR signaling, apoptosis, mammary gland development, and sexual dimorphism of liver gene expression. STAT5b deficiency is an extremely rare autosomal recessive disorder (10 reported cases) characterized by growth hormone-insensitive growth failure with intact growth hormone receptor, eczema, enteropathy, severe immunodeficiency, recurrent infections and chronic lung disease. We report a 13-year-old Kuwaiti female with STAT5b deficiency associated pulmonary alveolar proteinosis (PAP).

Design: A 13-year-old Kuwaiti female with STAT5b deficiency, dwarfism, and recurrent infections underwent a peripheral lung wedge biopsy secondary to pulmonary dysfunction. Tissue was obtained for routine processing, electron microscopy, viral and microbiologic studies, and cryopreserved for possible molecular genetics. Tissue was evaluated with routine, histochemical, immunohistochemical and electron microscopic techniques to evaluate for clinically suspected interstitial lung disease (ILD).

Results: Sections showed alveoli filled and distended by granular, eosinophilic proteinaceous material (PAS-positive and diastase-resistant). Numerous

cholesterol clefts and foamy macrophages (highlighted by CD68) were present within the material. Electron microscopy showed reactive type II pneumocytes with numerous typical surfactant lamellar bodies, and no surfactant deficiency or deficient transport features. Alveolar spaces were markedly distended by granular material containing occasional surfactant lamellar bodies and macrophages with frequent intracytoplasmic lamellar bodies. No microorganisms were identified by Silver, Gram, AFB, or Fite stains. CMV immunostaining and multiplex viral PCR were negative.

Conclusions: STAT5b deficiency is an extremely rare inherited disorder characterized by growth hormone-insensitive growth failure and severe immunodeficiency. PAP, a disease of intraalveolar surfactant accumulation secondary to impaired GM-CSF signaling in pulmonary macrophages, has been reported in association with antibodies against the GM-CSF receptor and, less commonly, with mutations in the genes encoding the GM-CSF receptor and surfactant proteins $\mathrm{B}$ and $\mathrm{C}$, but not previously in association with STAT5b deficiency. ILD is a well-known complication of STAT5b deficiency, but its etiology is not well characterized. Our study suggests both a novel mechanism for the development of PAP and a potentially under-recognized cause of ILD in patients with STAT5b deficiency.

1880 Comprehensive Genomic Profiling of Pediatric Gliomas Identifies Hypermutated Tumors with Mutations in DNA Mis-Match Repair Genes

Adrienne Johnson, Laurie M Gay, Jo-Anne Vergilio, Julia A Elvin, James Suh, Garrett Frampton, Siraj M Ali, Vincent Miller, Philip Stephens, Jeffrey Ross, John Crawford, Shakti Ramkissoon. Foundation Medicine, Inc, Cambridge, MA; University of California San Diego, San Diego, CA.

Background: Pediatric brain tumors are the leading cause of death among childhood cancers in the United States. Given the morphologic overlap seen among pediatric low-grade (PLGGs) and high-grade gliomas (PHGGs), there remains a critical need to incorporate next-generation sequencing data to inform clinical diagnostic, prognostic and therapeutic decision-making.

Design: We performed comprehensive genomic profiling on 186 pediatric glioma patients, including 112 PHGGs and 74 PLGGs. The average patient age was 12 or 9.5 years for PHGGs and PLGGs, respectively. Using DNA extracted from 40 microns of 
FFPE tumor sections, we sequenced 315 cancer-related genes using a hybrid-capture, adaptor ligation based next generation sequencing assay. We calculated tumor mutation burden (TMB) as the number of somatic, coding point mutations and indels per megabase (Mb) (low: $<1-5$, intermediate: 5-20, high: $\geq 20$ mutations/Mb).

Results: Among PHGGs genomic alterations were identified in $97.3 \%(109 / 112)$ of specimens with an average of 4.0 genomic alterations per patient. TMB ranged from $<1$ to 581 , with a median of 2.7 . Approximately $7 \%(8 / 112)$ of PHGGs were classified as hypermutated with TMBs ranging from 48-581. Interestingly this cohort harbored mutations in DNA mis-match repair genes, including $M S H 6, M S H 2, M L H 1$ and $P M S 2$, which are associated with biallelic mismatch repair deficiency. The most common alterations detected were $T P 53, H 3 F 3 A$ and $N F 1$ mutations.

In PLGGs, we detected genomic alterations in $95.9 \%(71 / 74)$ of tumors, with an average of 1.7 genomic alterations per specimen. TMB ranged from $<1$ to 532 with a median of 1.8 across the PLGG cohort. $B R A F$ was the most frequently altered gene with $47.3 \%(35 / 74)$ of patients harboring a lesion and included $B R A F$ V600E mutations, $B R A F$ fusions as well as one tumor with both a KIAA1549-BRAF fusion and $B R A F$ amplification. We detected other previously reported PLGG alterations including $F G F R I$ missense mutations and kinase domain duplications in $17.6 \%(13 / 74), N F 1$ loss of function mutations in $8.1 \%(6 / 74)$, and PIK3CA missense mutations in $6.8 \%(5 / 74)$. Conclusions: Comprehensive genomic profiling revealed genomic alterations in pediatric gliomas that can support diagnostic and therapeutic decision-making. Furthermore, we identified a cohort of hypermutated pediatric GBMs with high TMB and mutations in DNA mis-match repair genes. These findings suggest that TMB could be used as a biomarker to identify a subset of pediatric GBM patient who could potentially benefit from immunotherapy.

1881 Quantification of Colonic Mucosal Calretinin Immunostain in Hirschsprung's Disease (HD) Pull-Through Specimen by Image Processing and Analysis (IPA) Shows Substantial Variation and Limits Its Utility in Preoperative Mapping of Transition Zone

Israel Kasago, Sangtae Ahn, Sanaz Ainechi, Chunlai Zuo, Suzanne Homan, Christine E Sheehan, Hwajeong Lee. Albany Medical College, Albany, NY.

Background: Calretinin immunostaining of mucosal nerve fibers is used as a surrogate marker of submucosal ganglion cells in the evaluation of HD. We hypothesize that the amount of mucosal nerve fibers increases gradually in the transition zone (TZ) from an absence in the aganglionic zone (AZ) to uniform density in the normal zone (NZ). If so, preoperative mapping biopsies would define a $\mathrm{TZ}$ and eliminate the need for intraoperative frozen sections. Mucosal calretinin immunostain was quantified in the colonic mucosa from HD pull-through specimens by IPA.

Design: Calretinin immunostain was performed on representative cross ( 5 cases) and longitudinal ( 10 cases) sections from 15 HD pull-through specimens. The beginning of the $\mathrm{TZ}$ was defined as the point at which mucosal calretinin stained nerve fibers are first detected. The immunostained slides were scanned, and multiple images (mean 29 per case; range 13 to $56 ; 200 X$, jpeg format) of the colonic mucosa were taken from the proximal AZ, TZ and NZ. The calretinin stained areas in the mucosa were quantified using a pixel count technique by IPA that was developed in-house. The pixel count was plotted against the distance from the beginning of the $\mathrm{TZ}$.

Results: The mean length of the AZ was $7.1 \mathrm{~cm}(0.5$ to $15.6 \mathrm{~cm})$. The mean distance from the beginning of the $\mathrm{TZ}$ to the proximal resection margin (probable NZ) was 5.7 $\mathrm{cm}(1.7$ to $13 \mathrm{~cm})$. The mean of the amount of the calretinin stain per cross section increased from distal to proximal whereas the amount of the stain varied around the circumference in the same cross section. In longitudinal sections, the amount of the mucosal calretinin stain gradually increased from the beginning of the $\mathrm{TZ}$ towards the proximal resection margin, with positive correlation coefficients in 9 of 10 cases $(p<0.05)$. However, the variation of the staining in the probable NZ (at least $4.0 \mathrm{~cm}$ proximal from the beginning of the TZ) was large, with the mean coefficient of variation of 0.36 (range 0.18 to 0.87 ). No mucosal calretinin staining was noted in AZ.

Conclusions: By IPA, the amount of the mucosal calretinin staining gradually increases in the TZ in HD pull-through specimens. However, substantial variation of staining around the circumference at the same distance and in the NZ appears to limit the utility of quantification of mucosal calretinin staining as a mapping tool in defining the $\mathrm{TZ}$ and NZ in preoperative endoscopic mucosal biopsies.

\section{Russell Body Gastritis in Children: Histopathological Analysis} and Clinical Significance

Jaswinder Kaur, Hend Abulsayen, Siraj M El Jamal, Ali G Saad. University of Mississippi Medical Center, Jackson, MS

Background: Russell Body Gastritis (RBG) is a rare inflammatory condition characterized by abundant intramucosal polyclonal plasma cells containing intracytoplasmic eosinophilic globules of immunoglobulins (Russell bodies) that displace the nucleus. The etiology is unknown. Adult cases of RBG have been reported in associated with chronic gastritis, H. pylori organisms, HIV infection, and chronic inflammatory disorders including autoimmune disease such as rheumatoid arthritis. To the best of our knowledge, RBG in children has not been described. Here we report the histopathological and clinical association of 7 cases of RBG in children.

Design: Pathology systems were searched for chronic gastritis with Russell Bodies in children ( $\leq 18$ years of age). Slides were reviewed for the accuracy of the diagnosis. Clinical findings were retrieved from the patients' medical records. Immunostains for CD138, kappa, lambda and pankeratin were performed.

Results: The search resulted in 7 patients (4 males and 3 females; age range: $3.1-15.5$ years; average: 8.7 years). Four patients presented with isolated epigastric pain, 2 patients presented with vague abdominal pain and diarrhea, and one patient presented with nausea and vomiting predominantly post prandial. All patients underwent upper and lower gastrointestinal endoscopy and biopsy. Histologic examination of gastric biopsies showed numerous Russell bodies in gastric antral and fundic type mucosa associated with mild to moderate lymphocytic infiltrate. The plasma cells were positive for CD138 while kappa and lambda showed no restriction. Pankeratin was negative in all 7 cases. Follow up showed that 2 patients had an associated inflammatory bowel disease (Crohn's disease) in which chronic colitis with variable degree of activity was noted. Three patients were found to have H. pylori gastritis with no other abnormalities. In 2 patients, no other gastrointestinal diseases were identified, but these patients had a known history of juvenile arthritis.

Conclusions: RBG is poorly described in the pediatric population. Based on our case series, it appears that RBG is often associated with $\mathrm{H}$. pylori infection, chronic inflammatory bowel disease and rheumatologic disorders including juvenile arthritis. Immunohistochemical studies are necessary to document the polyclonality of plasma cells in these patients. Awareness of the morphological phenotype of RBG is necessary to avoid diagnostic pitfalls.

\section{Gestational Buprenorphine Therapy and Association with} Placental Dysmaturity

Elizabeth Kertowidjojo, Adela Cimic, Cynthia Kaplan. Stony Brook University Hospital, Stony Brook, NY.

Background: While methadone remains the standard of choice in opioid-substitution therapy during pregnancy, there is increasing interest in the use of buprenorphine, as it may reduce the incidence and severity of neonatal abstinence syndrome (NAS). Data on the effects of buprenorphine on fetal development is limited, and, to date, there are minimal reports describing the histologic changes in placental development associated with gestational buprenorphine therapy.

Design: Histologic evaluation was performed on three placentas from patients with known gestational buprenorphine use. Neonatal wellbeing was assessed by gestational age, Apgar scores, resuscitation, length of hospitalization and NICU stay, and presence of NAS.

Results: Case 1 is a $4080 \mathrm{~g}$ infant born at 39 weeks gestation with Apgar scores of 8 and 9 at 1 and 5 minutes after birth. Gestational buprenorphine dose was $1 \mathrm{mg}$ daily. The infant did not require any NICU stay or prolonged hospitalization. Case 2 is a $3820 \mathrm{~g}$ infant born at 40 weeks gestation with Apgar scores of 7, 8. Gestational buprenorphine dose was $12 \mathrm{mg}$ daily. The infant was diagnosed with NAS and required 6 days of NICU care. Case 3 is a $3510 \mathrm{~g}$ infant born at 37 weeks of gestation with Apgar scores of 7, 7. Gestational buprenorphine dose was $8 \mathrm{mg}$ twice a day and additional $4 \mathrm{mg}$ as needed. The infant was diagnosed with NAS and required 2 days of NICU care and 22 total days of hospitalization.

Placentomegaly was found in all three cases with placental weights of $740 \mathrm{~g}, 840 \mathrm{~g}$, and $840 \mathrm{~g}$, respectively. Placentas of all three cases also show dysmature chorionic villi with large irregular shapes, few syncytial knots, and increased vascularity with greater number of central vessels and reduced peripheral vessels. The degree of dysmaturity is focal in Case 1 and diffuse in Cases 2 and 3.

Conclusions: We present a report of three cases of placentomegaly and placental dysmaturity associated with buprenorphine therapy during pregnancy. The degree of enlargement and dysmaturity are correlated with higher doses of buprenorphine. This is an important observation, as surface area for oxygen diffusion is reduced in placentas with immature villi. Indeed, in these infants, villous immaturity was associated with lower Apgar scores, NAS, and prolonged hospitalizations. These cases also illustrate the importance of appropriate clinical history, as similar findings can be seen in other various conditions.

\section{4}

Mimics

PHOX2B Reliably Distinguishes Neuroblastoma from Histologic

John P Lee, Yin P Hung, Andrew M Bellizzi, Jason L Hornick. Brigham and Women's Hospital, Harvard Medical School, Boston, MA; University of Iowa, Iowa City, IA. Background: Neuroblastoma (NB), the most common solid extracranial pediatric tumor shows considerable histologic overlap with other small round blue cell tumors. While immunohistochemical (IHC) markers such as NB84 or synaptophysin have been used to help support the diagnosis of NB, they suffer from lack of specificity. PHOX2B, a transcription factor involved in the development of the peripheral autonomic nervous system, has recently been reported as an IHC marker for NB. However, this marker has not been studied in recently described round cell sarcomas, such as those with CIC-DUX4 rearrangement. The purpose of this study was to validate the specificity and diagnostic utility of PHOX2B for NB versus other small round blue cell tumors. Design: We evaluated 237 cases (130 in whole-tissue sections; 108 in tissue microarrays), including 73 NB [median age 2 yr; including 4 adult patients (29, 38, 62 , and 68 yr; 2 retroperitoneum, 1 adrenal, 1 thymus)] and 164 other tumors: 44 Wilms tumors, 20 Ewing sarcomas, $10 C I C$-DUX4 sarcomas, 10 poorly differentiated synovial sarcomas, 10 lymphoblastic lymphomas, 10 alveolar rhabdomyosarcomas, 10 embryonal rhabdomyosarcomas, 10 mesenchymal chondrosarcomas, 10 Merkel cell carcinomas, 10 olfactory neuroblastomas, 10 melanomas, 5 NUT midline carcinomas, and 5 desmoplastic small round cell tumors. IHC for PHOX2B was performed following pressure cooker antigen retrieval using a rabbit monoclonal antibody (clone EPR14423; 1:500 dilution; Abcam). Extent of nuclear staining was scored as 0 (negative), $1+(<5 \%$ cells positive), $2+(5-24 \%), 3+(25-49 \%), 4+(50-74 \%)$, or $5+(75-100 \%)$.

Results: PHOX2B was positive in 71 of $73(97 \%)$ NB, including all 69 pediatric cases but only 2 of 4 adult cases; 62 of 71 positive cases showed diffuse (at least $4+$ ) moderate-to-strong staining. Notably, only 4 of $164(2 \%)$ non-NB tumors tested showed any immunoreactivity for PHOX2B, including 2 Wilms tumors, 1 CIC-DUX4 sarcoma, and 1 Merkel cell carcinoma; all 4 showed minimal $(1+)$ weak-to-moderate staining. Conclusions: PHOX2B is a highly sensitive and specific IHC marker for NB. PHOX2B shows very limited staining in other small round blue cell tumors. PHOX2B reliably 
distinguishes NB from Wilms tumor, Ewing sarcoma, and CIC-DUX4 sarcoma, among other histologic mimics. PHOX2B negativity in 2 of 4 adult NB raises the possibility that some adult NB are of a different lineage than pediatric cases.

\section{Characteristics of Intestinal Metaplasia in Stomach Antrum in} a Pediatric Population

Geling Li, David R Kelly, Lei Zhao. Children's of Alabama, Birmingham, AL; Univ. of Alabama, Birmingham, AL.

Background: Gastric intestinal metaplasia (IM) is often viewed as precursor lesion for gastric adenocarcinoma in adults. Gastric antral IM is occasionally seen in children, but its clinical significance remains unclear. Here we set out to characterize the background histologic changes associated with gastric antral IM in the pediatric population.

Design: We retrieved biopsies from all patients 18 years or younger who had upper GI endoscopy with a pathologic diagnosis of IM in stomach antrum from 2003 to 2016. Follow-up biopsies for these patients were also collected. Each index and follow-up biopsy was evaluated for the presence of dysplasia, H. pylori, chronic gastritis and other pathologic changes.

Results: A total of 87 patients with antral IM were identified. Reviewing the index biopsies revealed 16 with chronic gastritis, 9 with chemical gastropathy, 5 with eosinophilic gastritis/infiltrates, and 9 with other pathologic changes ( 2 with focal active gastritis, 1 with fundic gland polyp, 1 with short bowel syndrome, and 5 with increased nonspecific chronic inflammation). The remaining 48 biopsies showed isolated IM with no other pathologic changes. Among the 48 patients, 7 patients had a clinical history of gastritis but showed no pathologic changes in the biopsy. No patients had H. pylori by routine or special stain.

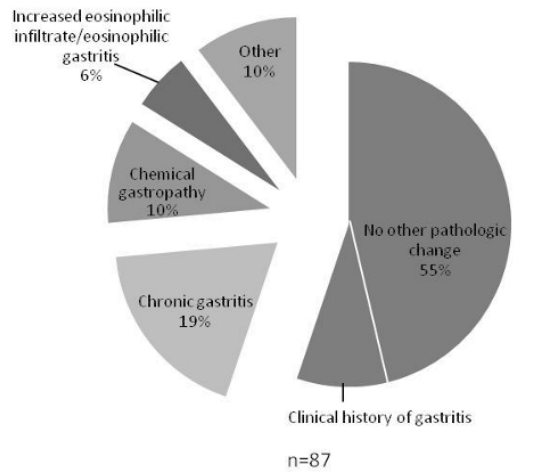

Sixteen patients ( 6 with chronic gastritis, 3 with eosinophilic gastritis/infiltrates, 7 with no other pathologic change) had undergone one or more repeat biopsy. Only 2 patients with eosinophilic gastritis showed persistent IM. None of the follow-up studies showed dysplasia or carcinoma.

Conclusions: The majority of antral IM in the pediatric population is an isolated finding with no other pathologic changes. Most of them likely represent sampling of transitional-type epithelium around the pylorus channel. A small percentage of isolated antral IM may be result of resolved gastritis. Chronic gastritis is the most common pathologic finding associated with antral IM; however, they are not associated with H. pylori infection as commonly seen in the adult population. Chemical gastropathy is the second most common finding. Eosinophilic gastritis or significant eosinophilic infiltrates seem to be associated with persistent antral IM in children.

1886 Congenital Leukemia: Clinicopathological Study of 5 Cases Amanda E Lo, Chuanyi Mark Lu, Rachel Jug, Catherine Luedke, Endi Wang. Duke University Medical Center, Durham, NC; University of California San Francisco, San Francisco, CA.

Background: Congenital leukemia in neonates and very young infants is rare. The risk, etiology, pathogenesis and prognosis of this neoplasm have not been fully understood. We report 5 cases of acute leukemia occurring within 60 days after birth.

Design: Medical records and pathologic specimens of 5 cases with congenital acute leukemia were retrospectively evaluated.

Results: Of 5 cases, 4 were male, 1 was female, 2 were Hispanic, 2 were AfricanAmerican, and 1 was non-Hispanic Caucasian. All patients were born full term with birth weight ranging from 2.9 to $3.5 \mathrm{~kg}$. Patients showed symptoms of leukemia at ages ranging from birth to 47 days, with a median age of 26.5 days. Patients came to clinical attention at ages ranging from 18 to 64 , with a median age of 32 days. Three patients presented with hepatosplenomegaly, 2 with bruising and petechiae, and 2 with severe anemia and tachypnea. Interesting, none of the patients presented with cutaneous involvement. Laboratory tests showed marked leukocytosis in all the cases, with a median white blood cell count of $483.5 \times 10^{9} / \mathrm{L}$ (range 240 - 1584.6). All patients had moderate to severe anemia/thrombocytopenia with a median platelet count of $38 \times 10^{9} / \mathrm{L}$. Numerous circulating blasts were present ranging from $80 \%$ to $99 \%$, with a median of $95 \%$ by manual differential count. Similarly, there were numerous blasts in the bone marrow with counts from $92 \%$ to $99 \%$, and a median of $93 \%$ blasts. The patient with the highest percentage of blasts in the bone marrow biopsy also showed central nervous system involvement. All 5 cases demonstrated immunophenotypic profiles diagnostic of B-lymphoblastic leukemia (B-ALL). Of note, 3 of 5 cases expressed CD10 and 2 of 5 cases were negative for TdT and CD34. Cytogenetic studies revealed clonal abnormalities with $M L L$ (11q23) gene rearrangement in all cases, including 3 cases with additional aberrations. All patents had combination chemotherapy, while 2 of the patients also received umbilical cord blood transplant. The median follow up period was 14.7 months (range 8.5 - 24) with all patients having died from disease progression.
Conclusions: All patients in our study presented with hyperleukocytosis in peripheral blood with high leukemic load in the bone marrow, and were diagnosed as B-ALL with $M L L$ rearrangement abnormalities. Though congenital leukemia patients often present with leukemia cutis, this was not seen in our series. Prognosis was dismal despite vigorous treatment.

1887 The Presence of Intervillous Thrombi Is Associated with Abnormalities in the Maternal and Fetal Circulation

Cathleen E Matrai, Rebecca Baergen. NYPH - Weill Cornell Medicine, New York, NY. Background: The etiology and significance of intervillous thrombi (IVT) is unclear. Though not exceedingly rare, a concrete association between these lesions and clinical disease states or fetal outcomes has not been reliably demonstrated. A recent report has shown a link between IVT and maternal diabetes (DM). Our study aims to evaluate the association of IVT with additional maternal conditions as well as placental pathologic findings.

Design: We identified 423 cases in our surgical pathology files. This included 317 cases with multiple IVTs and 106 gestational age-matched controls. The cohort was limited to term placentas ( $\geq 37$ weeks) and excluded multiple gestations, placenta accreta, maternal floor infarction, and those cases missing pertinent clinical data in both groups. Clinicopathologic features were retrospectively reviewed and statistical significance determined using Chi-squared and T-tests (Figure 1).

\begin{tabular}{|c|c|c|c|}
\hline \multirow{2}{*}{$\begin{array}{l}\text { Data } \\
\text { Clinical Data }\end{array}$} & \multicolumn{2}{|c|}{ Factor } & \multirow[t]{2}{*}{ P-value } \\
\hline & $\%$ IVT & $\%$ Controls & \\
\hline \multicolumn{4}{|l|}{ Maternal History: } \\
\hline Group B Streptococcus (GBS) & 9.8 & 21 & 0.004 \\
\hline Hypertension (chronic, gestational) & 10 & 12 & 0.59 \\
\hline DM (chronic, gestational) & 10 & 7.5 & 0.609 \\
\hline SLE & 1 & 1 & 0.580 \\
\hline Coagulopathy & 6.9 & 5 & 0.420 \\
\hline Post-Partum Hemorrhage & 1 & 1 & 0.363 \\
\hline Arrest of Descent & 12 & 18 & 0.068 \\
\hline Malpresentation & 5.3 & 1.9 & 0.104 \\
\hline Mode of Delivery, Cesarean Section & 63 & 52 & 0.035 \\
\hline Sex (\% males) & 54 & 53 & 0.329 \\
\hline Presence of Fetal Distress & 20 & 16 & 0.210 \\
\hline IUFD & 1.9 & 0 & 0.175 \\
\hline \multirow[t]{2}{*}{ IUGR } & 9.5 & 7.5 & 0.353 \\
\hline & IVT (mean) & Controls (Mean) & \\
\hline Maternal Age & 33.6 years & 34.3 years & 0.233 \\
\hline Birthweight & $3303 \mathrm{~g}$ & $3212 \mathrm{~g}$ & 0.131 \\
\hline Placental Weight & $459 \mathrm{~g}$ & $439 \mathrm{~g}$ & 0.088 \\
\hline Gravity & 2.4 & 2.6 & 0.415 \\
\hline Parity & 1.15 & 0.85 & 0.052 \\
\hline Aborta & 0.57 & 1.9 & 0.009 \\
\hline Apgar Scores ( 1 and 5 minutes) & $8.2,8.7$ & $8.3,8.8$ & $0.822,0.435$ \\
\hline Pathologic Data & $\%$ IVT & $\%$ Controls & \\
\hline Meconium & 52 & 46 & 0.41 \\
\hline $\begin{array}{l}\text { Uteroplacental Malperfusion (UPM) } \\
\text { (increased syncytial knots, accelerated villous } \\
\text { maturity, multifocal villous agglutination ) }\end{array}$ & 44 & 30 & 0.019 \\
\hline Infarcts & 19 & 25 & 0.467 \\
\hline Decidual Vasculopathy (DV) & 8.8 & 3.8 & 0.061 \\
\hline Retroplacental Hematoma (RPH) & 3 & $<1$ & 0.192 \\
\hline $\begin{array}{l}\text { Fetal Thrombotic Vasculopathy (FTV) } \\
\text { (thrombosis of fetal vessels, intimal fibrin } \\
\text { cushions, foci of avascular villi) }\end{array}$ & 13 & 6.6 & 0.021 \\
\hline Chronic Villitis & 9.1 & 9.4 & 0.306 \\
\hline Chorioamnionitis & 16 & 37 & $<0.005$ \\
\hline Funisitis & 8.5 & 16 & 0.015 \\
\hline $\begin{array}{l}\text { Umbilical Cord Abnormalities (insertion, } \\
\text { length, single artery, nuchal, knots) }\end{array}$ & 34 & 26 & 0.026 \\
\hline
\end{tabular}

Results: We found that the presence of FTV, UPM, umbilical cord abnormalities, and cesarean section were more common in the IVT group than in normal controls to statistically significant levels. The presence of chorioamnionitis, funisitis, GBS, and abortion were also statistically significant, but were all found to be less prevalent in the IVT group than in normal controls. DV and arrest of descent demonstrated an increased trend in the IVT group but did not reach statistical significance.

Conclusions: IVTs have been seen in association with fetomaternal hemorrhage and DM but their clinical significance remains unclear. We found IVTs to be more common in placentas with FTV, UPM, and umbilical cord abnormalities, suggesting that thrombosis in the intervillous space is associated with abnormalities in both the fetal and maternal circulations. Lack of association with diabetes may be due to the lower number of DM cases in our group relative to prior studies. The finding of decreased lesions in placentas with associated inflammatory/infectious pathology is not readily explainable and warrants further study, though may be attributed to a low sampling threshold for these patients. 
1888 Endoscopic Features and Clinical Symptoms Associated with Pediatric Helicobacter Infection

Nada Naiyer, Christina A Arnold, Michael A Arnold, Miriam R Conces. The Ohio State University Wexner Medical Center, Columbus, OH; Nationwide Children's Hospital, Columbus, $\mathrm{OH}$

Background: Dedicated comparison of endoscopic and histologic findings of Helicobacter pylori in the pediatric population has not been performed. As such, we evaluated endoscopic findings in pediatric $H$. pylori cases to correlate it with histologic findings.

Design: All H. pylori positive gastric biopsies were retrospectively collected from our primary and referral care center over twelve months (63 cases) and $120 \mathrm{H}$. pylori negative gastric controls were randomly selected from the same time period. Immunohistochemical staining for $H$. pylori was performed on all biopsies. Endoscopy reports and clinical symptoms from electronic records were reviewed and the findings were categorized and compared to the histologic findings.

Results: Within the reviewed 12 month period, 1,955 total pediatric gastric biopsies were identified, including $63 \mathrm{H}$. pylori positive cases $(3.2 \%)$. Normal endoscopic findings were significantly associated with $H$. pylori negative biopsies (cases $=20.6 \%$ vs. controls $=57.5 \%, \mathrm{p}<0.0001)$. Endoscopic nodularity was significantly associated with $H$. pylori infection (cases $=46.0 \%$ vs. controls $=5.8 \%, \mathrm{p}<0.0001$ ), while all other endoscopic features (erythema, gastritis, granular, cobblestoning, congestion, hemorrhage, erosions, edema, friable, and gastroparesis) and reported clinical symptoms were not significantly associated with $H$. pylori infection. Histologically, endoscopic nodularity was significantly associated with chronic inflammation, active inflammation, superficial inflammation and germinal centers. We previously demonstrated that germinal centers, active inflammation, moderate to severe chronic inflammation in the body, or chronic inflammation in the antrum were seen in each $H$. pylori positive biopsy. Among $H$. pylori positive cases, $13 / 63(20.6 \%)$ were suspected to have $H$. pylori based on endoscopic findings, while $13 / 63(20.6 \%)$ were endoscopically normal.

Conclusions: Endoscopic findings are a poor predictor of pediatric $H$. pylori infection. In H. pylori positive cases, suspicion for $H$. pylori is as common as a normal endoscopic impression. Our previous data demonstrates that histology shows superior sensitivity for pediatric $H$. pylori infection compared with endoscopic findings.

1889 Lobular Capillary Hemangioma of the Middle Ear in Children: Clinical, Pathological and Molecular Characteristics of Three Cases of This Exceedingly Rare Entity

Tejal Patel, Steven Andreoli, Siraj M El Jamal, Ali G Saad. University of Mississippi Medical Center, Jackson, MS; Wolfson Children's Hospital, Jacksonville, FL.

Background: Lobular capillary hemangioma (LCH) is an acquired benign vascular tumor of unknown origin. When it occurs in the head and neck region, it usually involves the skin and mucous membranes of the oropharynx. We report the clinical, radiological, histological and molecular characteristics of $3 \mathrm{LCH}$ involving the middle ear of children. Extensive review of the literature shows that these are the very first 3 cases of LCH occurring in the middle ear in the pediatric population.

Design: Three pediatric patients (case 1: 9.5 years/M; case 2: 12.5/M years; case 3: $14 /$ F years) presented with recurrent otitis media, facial paralysis and hearing loss, and ear pain. MRI (patient 2) revealed an avidly enhancing lobulated mass extending to the epitympanum and encasing the middle ear ossicles. The other 2 patients showed similarly enhancing lobulated tumors involving the middle ear. Excisional biopsy was performed on all 3 patients and tissue submitted for histopathological work up. Immunostains with CD31 and Glut-1 was also performed.

Results: Histological examination in all three patients was similar. Light microscopy showed capillaries lined with plump endothelium and pericytes. A certain disarray of endothelial cells was noted but no atypia or mitoses seen. The vessels were either round discrete or form a network. Delicate collagen separated the vessels and the former contained scattered inflammatory cells. The endothelial cells were positive for CD31 and negative for Glut-1. The histomorphology and immunoprofile were those of LCH; however, given the unusual location of these tumors, molecular confirmatory studies (FISH) which were performed on 2 patients and showed activating RAS mutations including NRAS confirming the diagnosis of $\mathrm{LCH}$. Clinical follow-up showed that case 1 is still disease-free, case 2 showed recurrence after 6 months and repeat surgery revealed a classic LCH with no atypical features. Case 3 had recurrence at 11 months but is still symptoms-free.

Conclusions: We describe in details 3 cases of $\mathrm{LCH}$ of the middle ear in children. The tumor recurred in 2 patients only few months after surgery. LCH should enter in the differential diagnosis of tumors of the middle ear in children. It appears that LCH of the middle ear have a tendency to recur necessitating close follow up.

1890 Pediatric Malignant Neoplasms Involving Horseshoe Kidneys: An Institutional Experience Including the First Case of Clear Cell Sarcoma of Kidney and the First Reported Case of EGFR Internal Tandem Duplication

Teresa Santiago, Michael R Clay, Elizabeth Azzato, Scott Newman, Israel FernandezPineda, Kim Nichols, Jinghui Zhang, James R Downing, Andrew M Davidoff, Rachel Brennan, David Ellison. St. Jude Children's Research Hospital, Memphis, TN.

Background: Horseshoe kidney (HSK) is one of the most common developmental disorders of the genitourinary system with an estimated incidence of 1 in 400 individuals. Neoplasms arise in approximately $12 \%$ of cases, with renal cell carcinoma, urothelial carcinoma and Wilms tumor (WT) being the most common. Although there is an increased incidence of WT in patients with a HSK, this structural abnormality is identified in less than $0.5 \%$ of all confirmed cases of WT. Clear cell sarcoma of kidney (CCSK) is far rarer pediatric renal neoplam than WT ( $<5 \%$ versus $87 \%$ overall), and is previously unreported in the setting of HSK. In this study, we explore the relationship between HSK and renal tumors in pediatric patients and report not only the first case of CCSK arising in HSK, but also the first reported case of epidermal growth factor receptor (EGFR) internal tandem duplication (ITD) in a CCSK.

Design: The medical records of children with neoplasia related to HSK (diagnosis between 1990 and 2016) were retrospectively reviewed for clinicopathologic data. Whole genome sequencing, whole exome sequencing, and transcriptome sequencing were available for one case.

Results: Six patients ( 3 female and 3 male; median age at diagnosis, 31.6 months) were identified. Of these, 5 patients had WT and 1 patient a CCSK. Tumor size ranged from 6.0 to $18.0 \mathrm{~cm}$ (mean, $12.4 \mathrm{~cm}$ ). At presentation, 4 patients were stage III, 1 patients was stage II, and 1 patient was stage I. Follow-up data were available for 5 patients; 4 patients with WT are alive with no evidence of disease (mean follow up, 11 years) and 1 patient with CCSK is still undergoing treatment. Combined next generation sequencing of the CCSK showed an ITD in the EGFR tyrosine kinase domain (TKD, exons 18-25). High levels of EGFR protein expression were confirmed by immunohistochemistry. Conclusions: In this retrospective study of six patients with HSK and a renal neoplasm, the most common neoplasm ( 5 of 6 ) was WT. The outcome and prognostic indicators for WT in a HSK and normal kidney are considered to be the same. In the remaining case, a CCSK is reported for the first time in HSK. This tumor harbored an ITD of the EGFR TKD. EGFR overexpression has been described in CCSK; however, this is the first report of an ITD of the EGFR TKD. The prominence of EGFR alterations in CCSK has implications for therapeutic approaches to this disease.

\section{TLE1 Immunohistochemistry, a Useful Adjunct in the Diagnosis} of Clear Cell Sarcoma of the Kidney

Jared T Shows, Ali Nael, Henry Tran, Ramzi Bawab, Steven Swartwood, Shengmei Zhou, Shamlal Mangray, Nick Shillingford. Children's Hospital Los Angeles, Los Angeles, CA; Keck School of Medicine, Los Angeles, CA; Rhode Island Hospital, Providence, RI; Warren Alpert Medical School of Brown University, Providence, RI.

Background: Clear cell sarcoma of the kidney (CCSK) is a rare malignant neoplasm that primarily occurs in children. It comprises approximately $3 \%$ of all malignant renal tumors in this age group. The tumor is histologically variable and can show morphologic overlap with small round blue cell tumors, in particular nephroblastoma (Wilm's tumor). Although CCSK lacks a specific immunophenotype, cyclin D1 has emerged as a useful immunohistochemical marker in the work-up of CCSK. TLE1 immunohistochemical (IHC) stain was initially touted as a robust marker for synovial sarcoma but further studies have demonstrated positivity in neurofibromas, schwannomas, malignant peripheral nerve sheath tumors, malignant mesothelioma and solitary fibrous tumor among others. We have encountered TLE1 IHC staining in CCSK so we investigated its utility in a series of cases.

Design: TLE-1 IHC staining was performed on a series of 12 CCSKs from 9 males and 3 females, aged 1 to 19 years. Seven tumors involved the right kidney and five the left. Results: All cases demonstrated a classic histology characterized by sheets, nests and cords of small neoplastic cells containing small round nuclei with fine chromatin and indistinct nucleoli, embedded in a vascular rich myxoid background. Cytoplasmic membranes were indistinct and mitotic figures were rare. Strong diffuse nuclear expression of TLE1 (figure 1) was present in 12/12 (100\%) cases.

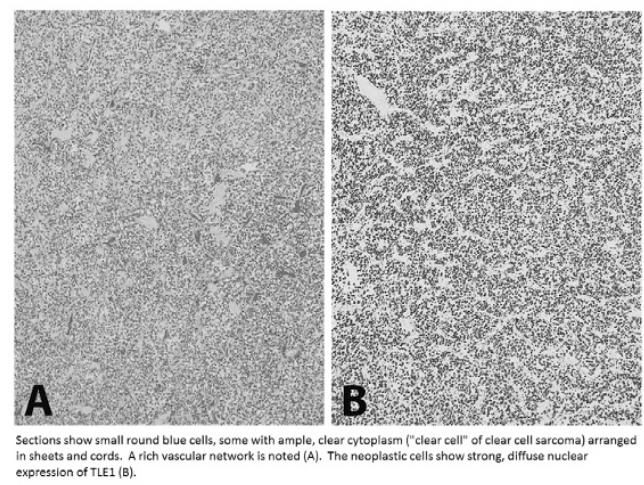

Conclusions: While not specific, TLE1 IHC can serve as a useful adjunct in establishing the diagnosis of CCSK. The results expand the list of tumors showing positivity for TLE1.

1892 Clinicopathologic Characteristics of Two Cases of Pediatric Histiocytic Neoplasms Following T-ALL with Identical Molecular Signature as Their Respective Original Malignant Clone

Catherine I Socec, Dehua Wang, Richard McMasters. Cincinnati Children's Hospital Medical Center, Cincinnati, OH.

Background: Histiocytic lesions have been reported in association with various hematopoietic malignancies, and rare cases developing after treatment for ALL might arise in similar if not identical clonal gene rearrangements. Here we discuss two cases of pediatric T-ALL with subsequent development of histiocytic neoplasms that proved deadly. The histiocytic neoplasms had the exact molecular signature of the patients initial T-ALL.

Design: We conducted molecular studies on both neoplasms, in each patient, to characterize the association with the original leukemia. We also compared original risk stratification of each patient, their therapies and their respective short latency periods to developing their fatal second neoplasm. 
Results: We show identical molecular signature in the original leukemic blasts and the subsequent histiocytic neoplasms in two pediatric patients with TALL. Using FISH, one case had the same biallelic deletion of the CDKN2A (TP16) locus on 9p21, while using next generation sequencing, the other had the same G13D mutation in the NRAS gene. Conclusions: This confirms a common clonal origin and highlights the need to perform appropriate molecular studies in such patients to better understand the mechanism and etiology behind histiocytic neoplasm seemingly born out of other hematopoietic malignancies.

1893

Assessment of PD-L1 Expression in Pediatric High-Grade Gliomas

Mariona Suñol, Iban Aldecoa, Ofelia Cruz, Angel Montero, Eva Rodríguez, Teresa Ribalta. Sant Joan de Déu Barcelona Children's Hospital, Barcelona, Spain.

Background: Programmed death-ligand 1 (PD-L1) is an immune-inhibitory receptor expressed in some tumors, including high-grade gliomas (HGG). Expression of PDL1 has been associated with poorer outcomes and may predict response to anti-PD-1 agents. Immune checkpoint-inhibitor therapies can facilitate tumor regression and clinical studies of PD-1 blockade are now being initiated in pediatric patients with high-grade gliomas (HGG), however, little is known regarding PD-L1 expression in these childhood tumors. In the present study, we aimed to investigate the expression of PD-L1 in a series of pediatric HGGs of our institution.

Design: We measured the incidence of PD-L1 expression in 54 pediatric HGGs (ages 0-17 y. o.). Whole slide sections were evaluated by IHC using the rabbit anti-PD-L1/ CD274 (clone SP142) monoclonal antibody. Nineteen of the tumors were autopsy cases. Tumor locations included pons (23), cerebral hemispheres (12), supratentorial deep structures (7), spinal cord (4), and other (8). The percentage of PD-L1-expressing cells was assessed semi-quantitatively.

Results: Fifty-seven percent (34/59) of tumors (28/40 biopsies; 6/19 autopsies) expressed PD-L1 in at least $1 \%$ of tumor cells. Staining was heterogeneous, with the vast majority of the cells demonstrating both membrane and cytoplasmic staining. Small clusters of strongly positive tumor cells were observed in 7 cases; a faint diffuse cytoplasmic staining was seen in 7 cases and scattered positive cells were identified in 20 cases. Many of the scattered PD-L1 expressing cells had either the morphology of either normal neurons, or inflammatory cells. A combination of patterns was observed in 7 cases. Two cases showed a weak nuclear stain.

Conclusions: Similar to adult HGG that have been recently profiled for PD-L1 expression, expression in our series of pediatric HGG patients using anti-PD-L1/CD274 (clone SP142) is frequent, although is largely confined to a minority subpopulation of tumor cells.

\section{4 \\ Histopathologic Correlation with the Newly Defined "Term"} Placenta

Ashley N Vogel, Jordyn B Tumas, Dan de Cotiis, Amanda Roman, Joanna Chan. Thomas Jefferson University, Philadelphia, PA; Einstein Medical Center, Philadelphia, PA.

Background: Previously, uncomplicated "term" pregnancies were considered a homogeneous group defined as 37-41 weeks gestational age. However, neonata outcomes, especially respiratory morbidity, varied significantly within this time frame. In 2013, the American Congress of Obstetrics and Gynecology (ACOG) reclassified term pregnancy into early ( 37 0/7-38 6/7 weeks), full (39 0/7-40 6/7 weeks), late (41 0/7-41 6/7 weeks), and postterm ( $>420 / 7$ weeks) pregnancies to reflect these clinical differences. This study examines the correlation between this new stratification and placental pathology.

Design: From 2012-2016, 674 consecutive singleton placentas were evaluated for gestational age, placental weight, cord insertion, malperfusion, fetal/maternal vascular stasis, meconium, fetal/maternal inflammatory response and decidual vasculopathy. Statistical analysis used single and multiple variable ANOVA as well as single and multiple variable logistical regression based on gestational age. When stratified into early, full, and late term, data was evaluated using Chi-Square and Fischer exact test analysis on a contingency table basis.

Results: Retrospective evaluation shows that placental weight $(\mathrm{p}<0.0003)$ and increased perivillous fibrin $(\mathrm{p}<0.001)$ directly correlate to gestational age. In addition, acute chorioamnionitis and funisitis correlate to increasing gestational age when compartmentalized as previously described ( $p<0.023$ and $p<0.001$, respectively). The remainder of the tested variables show no significant difference between early, full and late term placentas.

Conclusions: Stratifying placentas by the new ACOG terminology significantly correlates to placental weight, increased perivillous fibrin, chorioamnionitis and funisitis. The correlation between placental weight and gestational age is consistent with improved clinical outcome at increased gestational age. However, increased perivillous fibrin, chorioamniionitis and funisitis are each independently associated with poorer neonatal outcomes. Exact gestational age should be noted when evaluating placentas as it correlates with pathologic findings. Clinicians should be made aware of these risk factors as they advise their gravid patients. Future studies include correlating neonatal outcomes and maternal comorbidities with placental pathology in early, full and late term pregnancies.
1895 Norovirus Infection in Pediatric Small Intestine Allografts: A Clinicopathological Study of a Cohort of 23 Patients

Wei Xu, Stuart Kaufman, Joeffrey Chahine, Brandi Higgins, Nada Yazigi, Cal Matsumoto, Khalid Khan, Bhaskar VS Kallakury. Medstar Georgetown Univeristy Hospital, Washington, DC

Background: Human Norovirus in the family Caliciviridae is a major cause of epidemic gastroenteritis. Norovirus infections are typically acute and self-limited. However, Norovirus infection, the most frequent cause of acute pediatric gastroenteritis in the era of Rotavirus vaccine, produces a prolonged and chronic diarrhea in immunocompromised host, particularly in pediatric small intestine transplant recipients. This study aimed to characterize histological and immunohistochemical features of small bowl allograft biopsies before and after Novovirus infection.

Design: We retrospectively reviewed H\&E slides and performed IHC staining for proliferation index (Ki67), apoptosis (Caspase 3), T lymphocytes (CD3) on pre and post Novovirus enteritis (11 to 204 days from symptom onset to PCR diagnosis) in 23 small bowel transplant recipients at MGUH between 2007 and 2014. We studied immunohistochemical features by counting the number of intraepithelial $\mathrm{T}$ lymphocytes, proliferation index and apoptotic bodies in both villi and crypts per 100 enterocytes. Results: Comparison was made between pre Novovirus infection group (controls) and PCR confirmed Novovirus infection group. Compared with controls, biopsies with Novovirus infection group showed significantly increased in intraepithelial T lymphocytes $(15.1 \pm 6.0$ versus $7.7 \pm 2.9$ cells/100 enterocytes, $\mathrm{P}<.05)$, higher proliferation index in villi $(7.3 \pm 4.0$ versus $2.9 \pm 1.8$ cells $/ 100$ enterocytes, $\mathrm{P}<.05)$ and crypts $(78.7 \pm 12.1$ versus $50.3 \pm 11.7$ cells $/ 100$ enterocytes, $\mathrm{P}<.05)$, higher apoptosis activity in superficial lamina propria $(1.0 \pm 0.5$ versus $0.4 \pm 0.3$ cells $/ 100$ enterocytes, $\mathrm{P}<.05)$. There was no significant difference in crypts apoptosis activity between Novovirus infection group and control group.

Conclusions: In summary, all Novovirus infection biopsies showed increased intraepithelial $\mathrm{T}$ lymphocytes and villi blunting, increased proliferation index in both villi and crypts and increased lamina propria apoptosis, deemed characteristic of Novovirus infection. These features may be helpful in designing a test group to differentiate Novovirus enteritis from mild acute rejection, warranting further study.

\section{Pulmonary Pathology (including Mediastinal)}

1896 MET Exon 14 Splicing Mutations and Intragenic Deletions in Non-Small Cell Lung Cancer: A Study of Co-Occurring Genomic Mutations and Copy Number Alterations

Deepu Alex, Joseph Montecalvo, William Travis, Maria E Arcila, Marc Ladanyi. Memorial Sloan Kettering Cancer Center, New York, NY.

Background: Splicing mutations of $M E T$ exon 14 at the splice acceptor and donor sites have been previously reported to cause exon skipping, resulting in loss of the CBL E3ubiquitin ligase-binding site which is responsible of MET protein turnover. The presence of these oncogenic mutations makes the tumor responsive to MET-targeted therapies. Design: Comprehensive cancer genomic profiling was performed using a hybridization capture-based next-generation sequencing (NGS) assay for targeted deep sequencing of all exons and selected introns (inclusive of $M E T$ ) of 410 key cancer genes in formalin-fixed, paraffin-embedded tumors. Bar-coded libraries from patient-matched tumor and normal samples are captured, sequenced, and subjected to a custom analysis pipeline to identify somatic mutations, copy number alterations and select structural rearrangements. Next generation sequencing results from 1682 NSCLCs were reviewed to identify cases with $M E T$ exon 14 alterations.

Results: $M E T$ exon 14 alterations were detected in 58 patients [male $=27$, female $=31$ ] with a median age of 72 years (44-89). Tumor morphologies were variable and included pleomorphic sarcoma, lung adenocarcinoma (lepidic, acinar and mixed patterns), squamous cell carcinoma and adenosquamous carcinoma. 42 cases had sequence variants at the splice donor site ( 6 not previously reported), 11 cases had sequence variants at the splice acceptor site and 5 cases had deletions within exon 14 that included the CBL E3ubiquitin ligase-binding site, Y1003 (3 not previously reported). Based on the analysis of 410 genes of the sequencing panel, MET exon 14 mutated NSCLCs frequently showed the following co-mutations: TP53 alterations (32\%), MDM2 and CDK4 amplifications (25 and 21\% respectively), and TERT alterations (21\%). Concurrent MET amplification (as determined by the hybrid capture NGS assay) involving the mutated allele was detected in 5 cases $(9 \%)$. One case with $C B L$ p.R420L was identified. No other known lung cancer driver alterations were identified in these cases, indicating strong mutual exclusivity with alterations in EGFR, KRAS, ALK, RET and ROS.

Conclusions: $M E T$ exon 14 alterations represent a distinct molecular subtype of NSCLC. Our study describes the spectrum of alterations identified in this region, including alterations not previously reported. The detection of these alterations is important as it allows the identification of patients who can be treated with already existing targeted therapies.

1897 The Development of Brain Metastasis in Lung Adenocarcinoma Is Associated with a High Rate of Tumor Necrosis in the Primary Tumor and the Selection of Cribriform and Solid Growth Patterns

Khaleel I Al-Obaidy, Fulvio Lonardo. Wayne State Univeristy/ Detroit Medical Center/ Karmanos Cancer Institute, Detroit, MI.

Background: In lung adenocarcinoma (LADC), growth patterns- as recently re-definedand necrosis have a well-established prognostic value, yet it is not known whether they may be specifically linked to the development of brain metastasis. 